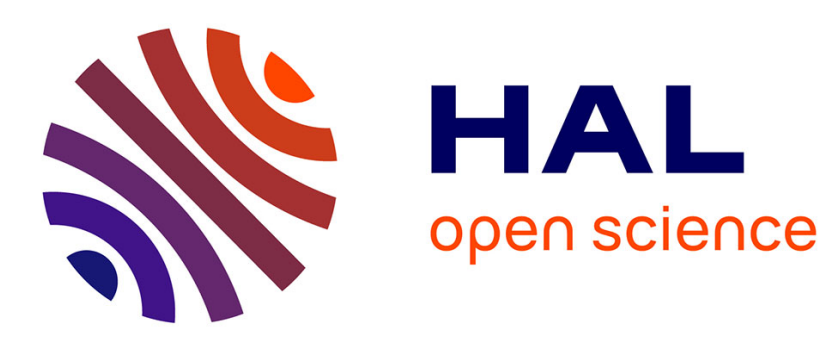

\title{
Generation of Pure OAM Beams with a Single State of Polarization by Antenna-Decorated Microdisk Resonators
}

Kévin G Cognée, Hugo M Doeleman, Philippe Lalanne, A Femius Koenderink

\section{- To cite this version:}

Kévin G Cognée, Hugo M Doeleman, Philippe Lalanne, A Femius Koenderink. Generation of Pure OAM Beams with a Single State of Polarization by Antenna-Decorated Microdisk Resonators. ACS photonics, 2020, 7, pp.3049 - 3060. 10.1021/acsphotonics.0c01081 . hal-03038304

\section{HAL Id: hal-03038304 \\ https://hal-iogs.archives-ouvertes.fr/hal-03038304}

Submitted on 3 Dec 2020

HAL is a multi-disciplinary open access archive for the deposit and dissemination of scientific research documents, whether they are published or not. The documents may come from teaching and research institutions in France or abroad, or from public or private research centers.
L'archive ouverte pluridisciplinaire HAL, est destinée au dépôt et à la diffusion de documents scientifiques de niveau recherche, publiés ou non, émanant des établissements d'enseignement et de recherche français ou étrangers, des laboratoires publics ou privés. 


\title{
Generation of Pure OAM Beams with a Single State of Polarization by Antenna-Decorated Microdisk Resonators
}

\author{
Kévin G. Cognée, Hugo M. Doeleman, Philippe Lalanne, and A. Femius Koenderink*
}

Cite This: ACS Photonics 2020, 7, 3049-3060

Read Online

ABSTRACT: We report waveguide-addressed plasmonic-photonic resonators that generate beams of controlled orbital angular momentum and spin angular momentum content upon driving through a waveguide. From phase-gradient metasurfaces, we borrow the idea of carefully combining multiple nanoscale resonators in a repeat unit, from which we build periodic rings that decorate microdisk resonators. We describe the general mode structure of microdisk cavities perturbed by antenna arrays on the basis of a quasinormal mode formalism and present a strategy to simultaneously control the orbital and spin angular momentum content
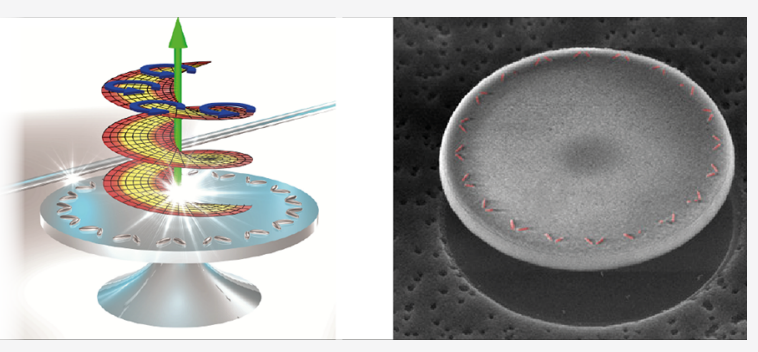
of light outcoupled to the far field. We propose a realization that uses silicon nitride disks and aluminum nanorod antennas. We find excellent polarization and orbital angular momentum (OAM) purity, as benchmarked by polarization-resolved interferometric Fourier microscopy on single devices.

KEYWORDS: localized plasmons, microcavities, orbital angular momentum beams, polarization control, hybrid plasmonic-photonic resonators, phase-gradient metasurface, Fourier microscopy

A ngular momentum has long been known to be carried by light, since the experiments and theory by Poynting and Beth $^{1,2}$ on the mechanical torque experienced by objects in circularly polarized beams. Further, both spin and orbital angular momentum have been known to appear in quantum mechanical selection rules since the early development of quantum mechanics. ${ }^{3}$ However, it is a seminal paper from Allen et al. in $1992^{4}$ that introduced the concept of generating and detecting pure orbital angular momenta of paraxial beams. For paraxial beams, the total angular momentum decomposes as spin angular momentum, i.e., circular polarization, and orbital angular momentum (OAM), which is purely ascribed to characteristics of the phase profile of the electromagnetic wave and not its polarization. ${ }^{4}$ OAM beams carry an optical vortex in their center, i.e., a zero in intensity around which the phase increments an integer number of $2 \pi$ (azimuthal dependence $\mathrm{e}^{i l \phi}$ for integer $\left.l\right) .^{5}$ The properties of light beams carrying OAMs can be used for various applications, ranging from imaging ${ }^{6-9}$ and micromanipulation, ${ }^{4,10-13}$ to freespace communication and quantum information. ${ }^{14-17}$ Furthermore, angular momentum is of large interest in nanophotonics, i.e., outside the limit of the paraxial approximation, where orbital and spin angular momentum degrees of light fields do not strictly separate. ${ }^{8,18-20}$ Interconversion between spin and orbital angular momentum by scattering off of nanoscale structures thereby ties into chiroptical scattering properties of handed scattering geometries and quantum mechanical selection rules in matter. ${ }^{20-23}$
To generate orbital angular momentum beams, researchers have on one hand reported lasers that intrinsically generate beams that carry OAM and, on the other hand, passive optical elements that transform a regular Gaussian beam. Since in this work we deal with a new strategy for generating OAM in a passive device, we refer the reader interested in lasers that generate OAM to the seminal review papers on $\mathrm{OAM}^{24,25}$ and henceforth focus purely on passive OAM generation strategies. For the transformation of a regular free-space homogeneously polarized Gaussian beam to one that carries OAM, traditionally spiral phase plates, q-plates (wave retarders with spatially varying birefringence axes), and liquid crystal spatial light modulators are inserted in the optical path. ${ }^{26-31}$ In recent years, OAM generation has also become a topic of investigation in nanophotonics, pushed by a growing interest in chiral plasmonic structures, ${ }^{21,22,32-35}$ and compact and broadband dielectric ${ }^{36-39}$ and metallic metasurfaces. ${ }^{40,41}$ Two main philosophies have been to replace spiral phase plates and spatial light modulators (SLMs) by metasurfaces, or alternatively to excite surface guided waves in metals that are outcoupled by geometrically spiralling structures. In a parallel development, researchers have been pushing for integrated

Received: July 6, 2020

Published: October 14, 2020 
devices whereby single mode waveguided input signals can be converted to send out free-space beams of specific OAM values, and conversely incident free-space beams of select OAM can be converted to on-chip signals. A number of works reported OAM generation by microdisk and microring resonators dressed by azimuthally periodic scattering corrugation. These include both OAM laser ${ }^{42-47}$ and waveguideaddressed passive devices. ${ }^{48-52}$

The main distinction to make in these efforts is between lasing devices on one hand, ${ }^{42-45}$ where one leverages the gain/ loss to select a single OAM value $l$ without its $-l$ counterpart, and waveguide-driven scattering devices on the other hand. $^{48-52}$ The common underlying physics is that distinct OAM orders are generated in direct relation to the number $N$ of repeating scattering units placed along the azimuthal direction in a WGM cavity mode of azimuthal mode number $m{ }^{48}$ analogous to the generation of discrete diffraction orders by gratings. In such schemes, a whispering gallery mode of azimuthal mode number $m$ dressed by $N<m$ corrugation scatters results in a superposition of right-handed circular polarization with OAM $l_{\mathrm{R}}=m-N+1$ and left-handed circular polarization carrying a different OAM $l_{\mathrm{L}}=m-N-1 .^{48}$

In this work we report devices that generate a single "pure" OAM state in a single circular polarization channel by waveguide excitation of a microdisk/microring cavity coupled to a chain of plasmonic antennas (Figure 1). The difference with the structures proposed in refs 42 and 50 is that purity is achieved without any a posteriori filtering by polarizing optics after generation by the microresonator. We leverage unit cell engineering, placing multiple plasmonic antennas in the unit cell of a periodic pattern that builds a ring of corrugations on the microdisk cavity. Tailoring the orientation, placement, and polarizability tensor of antennas within a unit cell provides control over the phase, amplitude, and polarization of outcoupled light, in the spirit of plasmonic metasurface physics, but confined to antennas on a ring. We experimentally demonstrate a simple realization of two different antennas per unit cell, where each unit cell acts as a source of circularly polarized radiation. An alternative interpretation is that while each antenna emits into both the right- and left-handed circular polarizations, one of the two channels is canceled by destructive interference while the other is enhanced. This proposed design can therefore scatter a polarization-pure OAM state and, by reciprocity, could also be used for OAM-selective detection and demultiplexing. The design strategy has implications for on-chip generation of vector vortex beams.

The paper is structured as follows. First, we explain the general mode structure of microcavities perturbed by antenna arrays and show that OAM can be generated according to strict arithmetic selection rules that are independent of whether the antennas couple significantly with each other, and independent of the degree to which they perturb the cavity mode. This first section is provided as a didactic basis to understand the main results of our paper. Next we explain the strategy for the generation of beams carrying pure OAM in a single polarization channel by placing multiple antennas in the unit cell. Finally we present an experimental realization in a silicon nitride microdisk resonator platform and dressed by aluminum antennas, verifying our predictions using interferometric polarization-resolved back focal plane imaging.
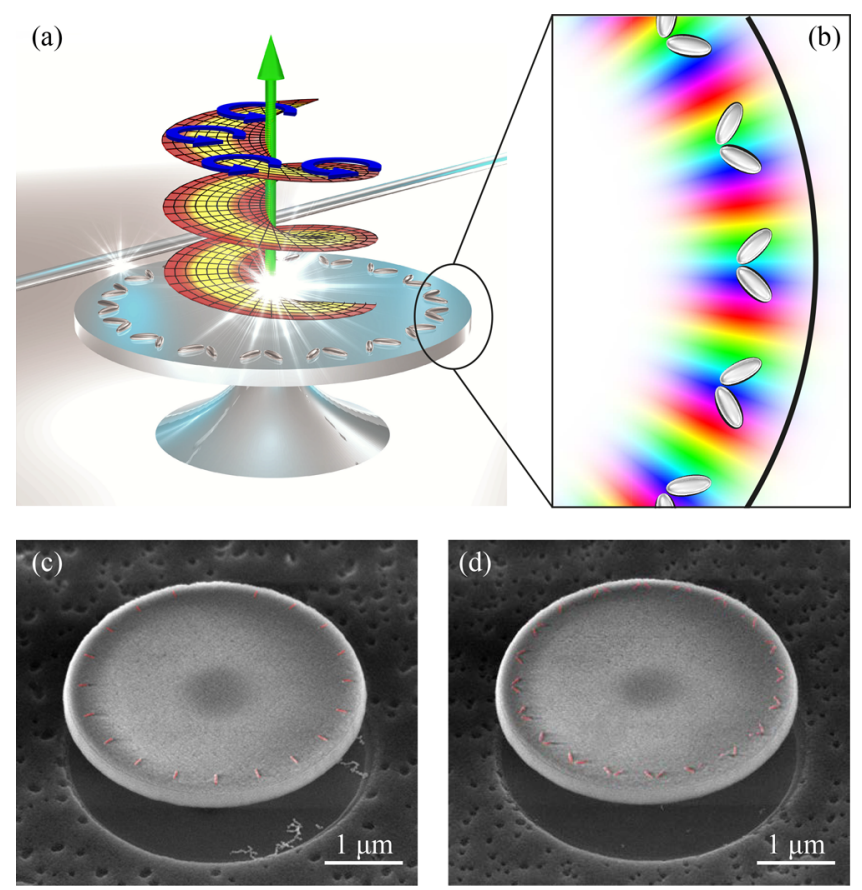

Figure 1. Sketch of our proposed design. A microdisk cavity dressed by a chain of plasmonic antenna and driven by light from a tapered fiber scatters a beam carrying orbital angular momentum (OAM). The design of the unit cell (inset) with pairs of antenna in a $\mathrm{V}$ or $\Lambda$ shape controls the scattering of a beam with OAM yet with pure circular-polarization. The color in the inset indicates the phase of the electric field of the cavity whispering gallery mode driving the antennas. (c, d) Electron micrograph of silicon nitride microdisks, covered with silicon oxide (glass), on top of which lie aluminum antennas: (c) radial-type structure with 20 antennas; (d) $\Lambda$-type (antenna pairs in $\Lambda$ orientation) structure with $2 \times 20$ antennas. We highlight the antennas in red for better readability. A glass shadow is visible under the disks as we used directional evaporation for the glass layer.

\section{MODEL}

This section aims to present the mechanisms by which the radiation pattern of a circular chain of antennas with a phase pattern varying as $\mathrm{e}^{i l \phi}$ contains orbital angular momentum. To this end, we first consider the general mode structure of microdisks that are decorated by antenna chains. Next, we review how the induced dipole moments yield far-field OAM. We build up from the far-field radiation patterns of continuous dipole current distributions on a ring to discretization of the current into $N$ segments. Next, we discuss polarization. Finally, we propose a design to engineer antenna arrays to obtain polarization-pure far-field orbital angular selection rules.

Modes of Disk Cavities Coupled to $N$ Antennas. Previously considered OAM generation by microdisks decorated with rings of antennas ${ }^{36,42,49,50}$ generally assumed the antennas to be nonresonant corrugations driven by whispering gallery modes of the disk, without considering coupling between the antennas among each other, or their perturbing influence on the disk modes. Here, we summarize the salient mode characteristics if one accounts for antennaantenna coupling through the cavity and for cavity perturbation by the antennas, thereby going beyond a mere classification of modes enabled by group theory. ${ }^{53-55}$ This perturbation analysis is based on quasinormal modes (see the Supporting Information and ref 56) and extends a significant 
body of literature on cavity perturbation and sensing. ${ }^{56-61} \mathrm{We}$ describe a chain of $N$ antennas (assumed identical, and identically oriented in a cylindrical coordinate frame) as $N$ polarizable dipole scatterers of polarizability $\alpha(\omega)$. Dipole $i$ in the chain is solely driven by the interaction with all the other particles in the chain $p_{i}=\epsilon_{0} \alpha(\omega) \sum_{j \neq i} E_{j \rightarrow i}$. Here, the fields $E_{j \rightarrow i}=\frac{\omega^{2}}{c^{2}} G\left(\mathbf{r}_{j}, \mathbf{r}_{i}, \omega\right) p_{j}$ are specified by a Green function ${ }^{62}$ (antenna coordinates $\mathbf{r}_{j}$, all antennas at distance $R$ from cylindrical axis, at angular positions $\left.\phi_{j}=2 \pi \frac{j}{N}\right)$. The full set of dipole moments is specified by a linear system

$$
\mathbf{M}(\omega) \mathbf{p}=\mathbf{E}_{\text {external }}
$$

with $\mathbf{p}=\left[p_{1}, p_{2}, \cdots\right]^{\mathrm{T}}$ and the interaction matrix $\mathbf{M}(\omega)$ of symmetric circulant form generated by the vector $\mathbf{c}=\left[\alpha^{-1}, g_{1}\right.$, $\left.g_{2}, \ldots, g_{2}, g_{1}\right]$, where $g_{n}(\omega)=-\frac{\omega^{2}}{c^{2}} G\left(\mathbf{r}_{i}, \mathbf{r}_{i+n}, \omega\right)$. Cylindrical circulant symmetry of the assumed cavity directly implies for any real or complex frequency, $\omega$, that $\mathbf{M}(\omega)$ has eigenvectors that carry OAM (integer $\left.k \in\left[\left[-\left\lfloor\frac{N}{2}\right\rfloor ;\left\lfloor\frac{N}{2}\right\rfloor\right]\right]\right)^{34,35}$

$$
\mathbf{v}_{k}=\frac{1}{\sqrt{N}}\left[1, \mathrm{e}^{i 2 \pi / N k}, \mathrm{e}^{i 2 \cdot 2 \pi / N k}, \ldots, \mathrm{e}^{i(N-1) 2 \pi / N k}\right]^{\mathrm{T}}
$$

with (degenerate) eigenvalues set by

$$
\begin{aligned}
\lambda_{k}(\omega)= & \alpha^{-1}(\omega)+g_{1}(\omega) \mathrm{e}^{i 2 \pi / N|k|}+g_{2}(\omega) \mathrm{e}^{i 2 \cdot 2 \pi / N|k|}+ \\
& \cdots+g_{1}(\omega) \mathrm{e}^{i(N-1) 2 \pi / N|k|}
\end{aligned}
$$

We refer to these eigenvectors as the antenna collective modes (or "supermodes") of index $k$. In order to obtain the coupled antenna-cavity eigenmodes, one needs to find complex frequencies $\omega$ for which $\lambda_{k}(\omega)=0$. At this point we focus on coupling of antennas to a specific pair of whispering gallery modes at azimuthal mode number $\pm m$. We assume that coupling to the cavity is sufficiently strong, such that antennas only couple to each other through the cavity mode. Since the Green function can always be decomposed on a cylindrical basis of Floquet modes, this approximation does not change the main conclusion on selection rules but allows the simplification of the Green function as

$$
g_{n}(\omega) \approx \frac{1}{\mu_{0} c^{2}} \frac{\widetilde{\omega}_{m} \tilde{E}_{m}^{2}}{\omega-\widetilde{\omega}_{m}} \cos \left(m \Delta \phi_{n}\right)
$$

with $\Delta \phi_{n}$ the angular spacing between an antenna and its $n$th neighbor. Here, $\widetilde{E}_{m}$ and $\widetilde{\omega}_{m}$ are the quasinormal mode profile and complex-valued eigenfrequency defined in ref 63, where $\tilde{E}_{m}$ is understood to be the radial component of the normalized (complex-valued) mode function as the azimuthal part is assumed to be negligible in our designs. ${ }^{56}$ Inserting this in $\lambda_{k}(\omega)=0, N$ antennas modes hybridize with two degenerate cavity WGM leading to the following mode structure.

(1) Out of $N$ antenna collective (or super-) modes, just a pair of degenerate modes is relevant as these modes are the only ones that can (weakly) hybridize with the pair of cavity WGM. This hybridization gives two high- $Q$ modes which are perturbed cavity modes, and two low$\mathrm{Q}$ modes which are perturbed antenna modes. Only the "cavity-like" modes are relevant in our work since they couple to the assumed fiber taper cavity input.
2. If $m$ is an integer multiple of $N$, the two cavity-like modes are standing wave eigenmodes and are not degenerate. Indeed, one of them is not coupled to antenna and therefore not perturbed since antennas are placed at nodes of its field, while the other is maximally coupled to the antennas (therefore shifted in frequency and perturbed in $Q$ ) since antenna are exactly at the antinodes of the field. The complex-valued frequency shift $^{57}$ is

$$
\frac{\Delta \widetilde{\omega}}{\widetilde{\omega}}=-N \alpha(\widetilde{\omega}) \tilde{E}_{m}^{2}
$$

i.e., $N$ times the frequency shift and damping rate change induced by a single antenna. ${ }^{56,57,64}$

3. If instead $N$ does not fit an integer number of times in $m$, the two counterpropagating cavity eigenmodes (OAM \pm $m$ ) remain degenerate, both shifted in frequency by

$$
\frac{\Delta \widetilde{\omega}}{\widetilde{\omega}}=-\frac{N}{2} \alpha(\widetilde{\omega}) \tilde{E}_{m}^{2}
$$

i.e., with each antenna contributing only half the shift. The cavity mode $\pm m$ is coupled only to antenna mode $\mp k$ if $m+k$ is divisible by $N$, and to $\pm k$ if $m-k$ is divisible by $N$.

The practical conclusion for OAM generation is that if the number of antennas $N$ is chosen not quite equal to $m$, then the cavity modes remain degenerate. When just one of the traveling modes is excited, e.g., by side-coupling to an adjacent waveguide, the antennas form a discrete ring of dipoles excited with a phase increment from antenna to antenna that corresponds to an orbital angular momentum $k$. An important assumption is that the perturbation in frequency is small compared to the frequency spacing between disk modes of different radial and azimuthal quantum number, so that mixing is avoided.

Continuous OAM-Carrying Scalar Current Distributions. Let us consider a ring $S$ of radius $R$ on which a current distribution $j(\mathbf{r})$ is imposed of constant amplitude, but carrying a pure phase $\mathrm{e}^{i l \phi}$ (with integer $l$ ) varying with the azimuthal position $\phi$ on the ring ${ }^{65}$

$$
j(\mathbf{r}) \propto \mathrm{e}^{i l \phi} \delta(z=0, r=R)
$$

In a scalar model, this current gives as far-field radiation pattern $F_{S}(\mathbf{k})$ (scalar far field) a Bessel beam with a single OAM contribution ( $\mathrm{k}$ momentum vector of radiated light)

$$
F_{S}(\mathbf{k}) \propto(-i)^{l} \mathrm{e}^{i l \psi} J_{l}(k R \sin (\theta))
$$

where $k=\|\mathbf{k}\|, \theta$ is the angle of $\mathbf{k}$ with the normal to the ring plane, and $\psi$ is the azimuth. Mathematically this procedure just states that the radiation pattern of a current reflects the Fourier transform of the current distribution. The ring radiates light according to a Bessel function of the first kind, with a phase distribution that varies as $\mathrm{e}^{i l \psi}$, i.e., as the imposed phase on the current distribution.

Discrete versus Continuous Distributions. A discrete current distribution of $N$ sources

$$
\begin{aligned}
j_{N}(\mathbf{r}) & =j(\mathbf{r}) \sum_{n=1}^{N} \delta\left(\phi=\frac{2 \pi}{N} n\right) \\
& \propto \mathrm{e}^{i l \phi} \delta(z=0, r=R) \sum_{n=1}^{N} \delta\left(\phi=\frac{2 \pi}{N} n\right)
\end{aligned}
$$


is simply the product of a continuous current distribution and a sum of delta-functions. This sum of delta-function is $\frac{2 \pi}{N}$ -periodic in the azimuthal direction and can be expressed as a Fourier series $\sum_{q=-\infty}^{+\infty} \mathrm{e}^{i q N \phi}$, leading to

$$
j_{N}(\mathbf{r}) \propto \sum_{q=-\infty}^{+\infty} \mathrm{e}^{i(l+q N) \phi} \delta(z=0, r=R)
$$

The discretized distribution is therefore equivalent to a superposition of continuous distribution at all OAM equal to $l$ modulo $N$. This can be viewed as similar to "aliasing" in discrete Fourier transforms: ${ }^{65}$ if one discretely samples a harmonic signal of order $l$ on $N$ points, its sampling is indistinguishable from that of any harmonic signal with $l^{\prime} \equiv l$ $\bmod N$. It is also analogous to the well-known case of a discrete grating that approximates a continuous phase distribution of in-plane wavevector $k_{\|}$, but which will radiate higher-order Fourier components as grating orders. The scattering of $N$ antennas driven by a field with azimuthal dependence $\mathrm{e}^{i l \phi}$ (angular momentum $l^{\prime}$ ) will similarly contain a sum of terms $J_{l^{\prime}}$ $(k R \sin \theta) \mathrm{e}^{i l^{\prime} \phi}$ with contributions of all orbital angular momentum of orders $l^{\prime}=l+q N$, with $q \in \mathcal{Z}$. In the following, we will refer to these terms as the fundamental OAM, and to the sidebands as higher-harmonic orbital angular momentum. For a high enough number of antennas, these higher harmonics only contribute far outside the paraxial direction.

Circular Polarization. Thus far, we have quoted results for scalar currents radiating scalar fields. The results directly transpose to radially and tangentially polarized dipole current distributions of OAM $l$, which will result in linearly radially respectively azimuthally polarized Bessel beams also of OAM $l$, and their higher harmonics. In this work, instead, we are interested in polarization-pure OAM output results but in other polarization channels, notably lab-frame left- and righthanded circular polarization. Dipole current distributions and beams of OAM $l$ can be decomposed in left-handed (LHCP) and right-handed (RHCP) components, however, not of OAM $l$ but $l \pm 1$. Figure 2 illustrates this well-known and purely geometrical effect. For instance, the transverse profile of a radially polarized beam of given OAM value $l$ can be written as $\mathbf{E}(\mathbf{x}, \mathbf{y}) \propto A(\rho) \mathrm{e}^{i l \phi}(\cos \phi, \sin \phi)$ with $A(\rho)$ a radial amplitude distribution, $\mathrm{e}^{i l \phi}$ the OAM-carrying phase-term, and (cos $\phi, \sin \phi)$ the radial unit vector. At azimuthal coordinate $\phi$, the electric field is polarized at an angle $\phi$ to the horizontal. Since a linear vector at arbitrary angle $\beta$ from the horizontal decomposes as linear superposition of left- and right-handed basis vectors that contribute with opposite phase factors $\mathrm{e}^{ \pm i \beta}$ (cf. Figure 2a), the additional phase dependences add up to the RHCP and LHCP phase profile and lead to the fact that the radially polarized beam of OAM $l$ actually decomposes in, respectively, $l+1$ and $l-1$ RHCP and LHCP contributions (Figure 2b). Instead of phrasing the argument for electric field polarization in a beam, one can also phrase it in terms of antenna polarization axis. This means that, for a cavity decorated by a ring of $N$ radially oriented antennas driven by a radial cavity field of OAM $l$, each antenna is equivalent to the superposition of a right- and a left-handed circularly polarized dipole radiator with excitation respectively proportional to $\mathrm{e}^{(l+1) \beta_{n}}$ and $\mathrm{e}^{(l-1) \beta_{n}}$, where $\beta_{n}$ is the azimuthal position (a)

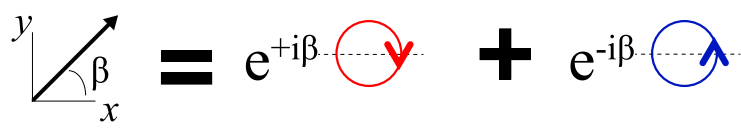

(b)

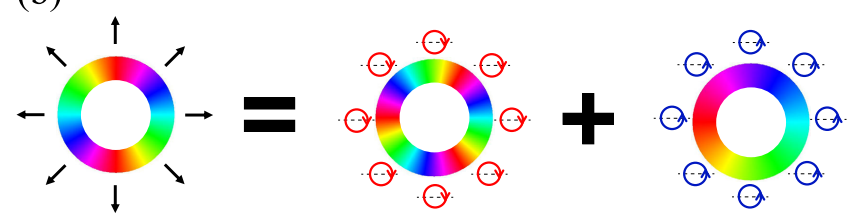

Figure 2. (a) A linear electric field vector oriented at angle $\beta$ relative to the $x$-axis decomposes as a superposition of left- and right-handed contributions that carry a phase $\pm \beta$, since in Cartesian coordinates $(\cos \beta, \sin \beta)=\left(\mathrm{e}^{i \beta}|L\rangle+\mathrm{e}^{-i \beta}|R\rangle\right) / \sqrt{2}$ with $|L, R\rangle=(1, \mp i) / \sqrt{2}$ the LHCP and RHCP Jones vectors. (b) A radially polarized beam that appears to carry OAM $l$ when the field is expressed in cylindrical coordinates (radial field $\propto \mathrm{e}^{i l \phi}$, yet azimuthal field component identically 0 ) decomposes as two opposite helicity beams that do not have OAM $l=2$ but $l_{\mathrm{R}}=l+1$ and $l_{\mathrm{R}}=l+1=+1$ for RHCP and LHCP, respectively, owing to the fact that the radial basis vector rotates around the beam axis.

of antenna $n$ and therefore also its orientation. Some authors view this result, which essentially comes from the basis transformation from cylindrical to Cartesian coordinates, as conservation of total angular momentum whereby the sum of OAM and spin (+1 for LHCP, -1 for RHCP) must be the same for both polarization channels ${ }^{20}$ for a source distribution with no intrinsic spin.

Generating Pure OAM in a Single Circular Polarization State. We now turn to the main idea of the paper, namely, to apply unit-cell polarizability tensor engineering to control output polarization, i.e., to generate labframe-polarization pure OAM beams. Regular arrays of radially polarized antennas driven by a single OAM (cavity WGM) are equivalent to a superposition of circularly polarized dipoles with opposite handedness and different OAM that will simultaneously scatter beams in both left and right circular polarization, but also with different OAM (and their harmonics). Inspired by the complex unit cells of phase gradient metasurfaces, ${ }^{66}$ one can incorporate multiple antennas in each unit cell and engineer the resultant superpositions of polarization by controlling the spacing and orientation of antennas within the unit cell.

We show that one can obtain a target OAM of value $l$ that is of pure circular polarization as the output beam using a whispering gallery mode of azimuthal mode number $m$ and a total of $N$ scattering unit cells such that $l=N-m$, but having two instead of one antenna per unit cell to obtain polarization control. The design is sketched in Figure 3, where essentially two interspersed arrays of $N$ antennas are placed on a ring, one array with antennas rotated by $+45^{\circ}$ from the radial direction, and the second rotated by $-45^{\circ}$. The two arrays are placed at the same radial distance from the center of the cavity, but azimuthally shifted by an angle $\Delta \phi$. The rationale of this design rests on the fact that when the linear polarization of a dipole current distribution is rotated by an arbitrary angle $\beta$ with the radial direction, there is a global phase slip between the right- and left-handed polarization, as shown in Figure 2a. Therefore, one can cancel out one of two circular polarizations 


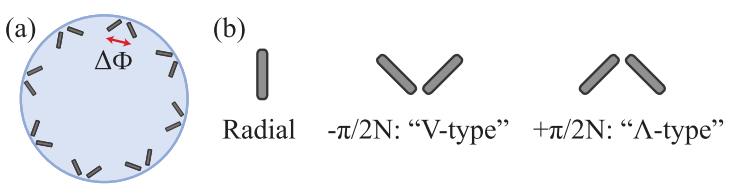

Figure 3. (a) Sketch of a disk dressed with $N$ pairs of antennas. In each pair, antennas are rotated by $+45^{\circ}$ and $-45^{\circ}$ from the radial direction and separated by an angle $\Delta \phi$ (center to center). (b) We propose three geometries for our experiment: radial orientation, $\mathrm{V}$ type, and $\Lambda$-type.

by interspersing two arrays, one with antennas rotated by $\beta=$ $+45^{\circ}$, and the other by $\beta=-45^{\circ}$, and engineering the phase delay introduced by the spatial offset $\Delta \phi$ for destructive interference between the two radiated fields.

The design proposed in Figure 3 is easy to analyze, assuming antennas are polarized by a cavity WGM of mode number $m$ (radial field varying as $\mathrm{e}^{i m \phi}$ ). For $N$ unit cells, one obtains the fundamental OAM $l=m+N$ and the harmonics $l^{\prime}=m+q N$, with $q \in \mathcal{Z}$, with the net dipole moment per unit cell decomposed in labframe right- and left-handed components as

$$
\left\{\begin{array}{l}
p_{R, l^{\prime}}(\phi) \propto \mathrm{e}^{i\left(l^{\prime}+1\right) \phi} \cos \left(\frac{\left(m-l^{\prime}\right) \Delta \phi}{2}-\frac{\pi}{4}\right) \\
p_{L, l^{\prime}}(\phi) \propto \mathrm{e}^{i\left(l^{\prime}-1\right) \phi} \cos \left(\frac{\left(m-l^{\prime}\right) \Delta \phi}{2}+\frac{\pi}{4}\right)
\end{array}\right.
$$

Focusing on the fundamental OAM harmonic only, in order to only have right (left)-handed light radiated into the far field, it suffices to remove the left (right)-handed source component by adjusting the antenna spacing $\Delta \phi$ to $(-) \frac{\pi}{2 N}$. This leads to so-called "V-type" structures with $\Delta \phi=-\frac{\pi}{2 N}$, and " $\Lambda$-type" structures with $\Delta \phi=+\frac{\pi}{2 N}$ (Figure 3) that, respectively, cancel right- and left-handed polarization. The choice of $\Delta \phi$ can be understood as requiring the two orthogonal dipoles in one unit cell to radiate with a $\pm \pi / 2$ phase difference, i.e., as a purely circular dipole. In more microscopic detail, the second dipole is $\pm m \frac{\pi}{2 N}$ delayed/advanced compared to the first inside the cavity mode profile, because the driving is delayed. Because it is also rotated by $\pm \frac{\pi}{2 N}$, it emits the OAM of $l=m+N$ with a phase advance of $\pm l \frac{\pi}{2 N}$, and therefore, the accumulated phase delay of the contribution of the second antenna compared to the first one is $\pm(m-l) \frac{\pi}{2 N}= \pm \frac{\pi}{2}$.

Predicted Radiated Fields. Figure 4 shows calculated radiation patterns that illustrate the proposed control over OAM and polarization purity. We calculate the far field radiated by the ensemble of $N$ unit cells (single dipoles or dimers) following the design rules for radial-type, V-type, and $\Lambda$-type structures. We account for the driving by a radially polarized cavity field of azimuthal number $m$ by fixing the driving of each dipole $i$ placed at the azimuth $\phi_{i}$ to $E_{i} \propto \mathrm{e}^{+i m \phi_{i}}$. We construct the radiation pattern by superposition of free space dipole radiation patterns, meaning that we ignore any modification that the cavity has on far-field radiation patterns of each single antenna, which is a reasonable assumption for cavities with cylindrical symmetry which does not perturb OAM content. We assume a radius of $2 \mu \mathrm{m}$ for the array of dipoles and present phase and intensity profiles of the scattered field in both circular polarization channels, obtained for $m=22$ and $N=20$ unit cells, for the radial-type, V-type, and $\Lambda$-type structures. Intensities in LHCP and RHCP are normalized to the maximum of the total intensity and follow a Bessel function dependence as predicted by eq 5 . The Bessel function changes sign when going through its radial nodes, thus explaining radial phase jumps of $\pm \pi$. Figure 4 clearly shows that the simple radial antenna structure scatters light into both helicity channels, whereas the $\mathrm{V}$ - and $\Lambda$-type structures favor, respectively, LHCP and RHCP. For all structures where the LHCP channel carries intensity, it exhibits a central phase
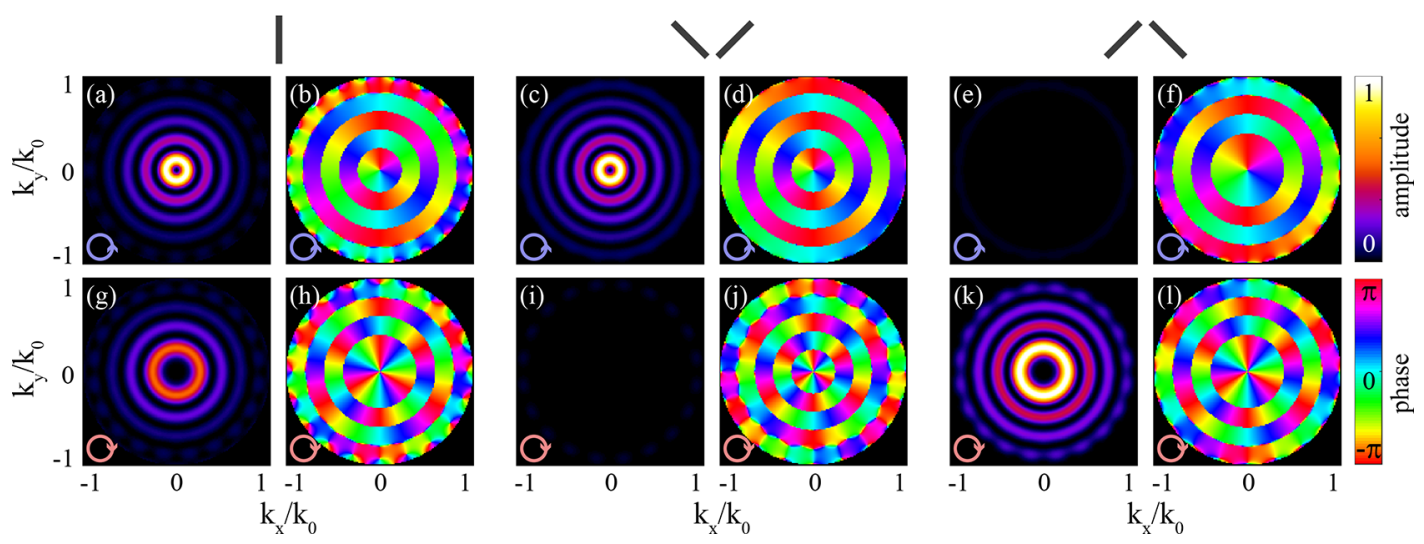

Figure 4. Calculated phase and intensity of radiated OAM beams for WGM mode number $m=22$ coupled to $N=20$ dipoles regularly positioned at a radius $2 \mu \mathrm{m}$. Panels a, b, g, and h ( 2 leftmost columns) correspond to a radial I-type structure; panels c, d, i, and j (middle columns) to a V-type structure; and panels e, f, k, and 1 (rightmost columns) to a $\Lambda$-type structure. Panels a-d (top row) are obtained for left-handed circular polarization (LHCP) and panels e-h (bottom row) for right-handed circular polarization (RHCP). For each structure and polarization channels, we plot the intensity (left image) and phase profile (right image). The intensity profile follows a Bessel dependence as predicted by eq 5 . The Bessel function changes sign pseudoperiodically, thus explaining radial phase jumps of $\pm \pi$ at the nodes' intensity profiles. We observe that, for the radial structure (a, g), both polarizations have similar intensity, whereas for the $\mathrm{V}$ - and $\Lambda$-type structures, respectively, left (c, i) or right (e, $\mathrm{k}$ ) polarization is favored. For all structure types, we observe in panels $\mathrm{b}$, $\mathrm{d}$, and $\mathrm{f}$ that the left polarized channel exhibits a central phase singularity corresponding to an OAM of $l_{\mathrm{L}}=m-N-1+1$, and in panels $\mathrm{h}, \mathrm{j}$, and 1 that the right polarized channel exhibits a central phase singularity corresponding to an $\mathrm{OAM}$ of $l_{\mathrm{R}}=m-N+1=+3$. 
singularity corresponding to an OAM of $l_{\mathrm{L}}=m-N-1=+1$, whereas RHCP exhibits an OAM of $l_{\mathrm{R}}=m-N+1=+3$ exactly as expected. The parasitic higher-order OAM that is due to the finite number of radiating unit cells can be observed in all phase profiles as phase and amplitude modulation in the azimuthal direction for the highest wave-vectors, commensurate with the fact that high OAM contributions correspond to high-order Bessel beam contributions.

\section{EXPERIMENTAL REALIZATION}

Design and Fabrication. We have realized the proposed design in a platform of $\mathrm{Si}_{3} \mathrm{~N}_{4}$ microdisks driven by a tunable narrowband laser through a tapered fiber and mounted in an interferometric Fourier microscope to observe the far-field scattering of antenna arrays that decorate the disks. The precise design parameters, i.e., disk diameter, radial positioning of the antennas, and the vertical spacing of the antennas to the disk through a low index spacer, have been optimized by finite element simulations. These parameters trade off optimum placement in the TE mode profile, decoupling from the TM modes and retention of appreciable $Q$-factors, and matching of the far-field pattern to the numerical aperture and resolution of our Fourier microscopy setup. The antenna material and dimensions were optimized to ensure a strongly anisotropic polarizability for each rod, in interest of the scattering contrast. Optimization results are given in the Supporting Information. The device fabrication (Methods section) resulted in $200 \mathrm{~nm}$ thick $\mathrm{Si}_{3} \mathrm{~N}_{4}$ disks with diameters in a small range around $4 \mu \mathrm{m}$ diameter to target $m \sim 20-25$ azimuthal mode numbers, with aluminum nanorod antennas lifted approximately $110 \mathrm{~nm}$ from the top of the disk to limit their effect on the cavity $Q$. The antennas are near-resonant with the cavity to allow for a large polarizability anisotropy $\left|\alpha_{\text {long }} / \alpha_{\text {short }}\right|^{2}>100$. Example devices are shown in Figure 1c,d.

We use tapered fiber excitation with a narrowband external cavity tunable diode laser and perform high-NA (numerical aperture, NA $=0.95$ ) phase-resolved Fourier imaging of the radiation pattern as shown in Figure 5a. To this end, we recombine on a CCD camera the Fourier image with an expanded reference beam to record an interferogram. By using an unbalanced interferometer, we can scan the phase delay over ca. $6 \pi$ in 200 steps by slightly detuning the laser frequency (see the Methods section). With the fabricated samples, the only fundamental radial TE mode in the range of our tunable laser $(\lambda=765-781 \mathrm{~nm})$ is $m=22$. This mode is always measurable for two different disk sizes in each set of 5 different sizes that we fabricated (target of 1.96, 1.98, 2.00, 2.02, and $2.04 \mu \mathrm{m}$ radii). We therefore interrogate devices with $N=19-$ 23 unit cells of radial-type, $\mathrm{V}$-type, and $\Lambda$-type structures, thereby expecting to generate OAM -2 to +4 . Disks without any antenna have a quality factor $Q \approx 2 \times 10^{4}$, which drops to around 500-1000 with antennas (ca. $1 \mathrm{~nm}$ line width).

Figure 5 documents the measurement protocol on a single device with $N=19$ scattering unit cells. As a first step, we record the intensity profile of the analyzed beam (Figure $5 b$ ) and the reference beam (Figure 5c). Then, we record a set of 200 interferograms over a total $6 \pi$ phase increment (examples shown in Figure $5 \mathrm{~d}, \mathrm{e}$ ). The radiation pattern shows a ring type structure, with the main lobe at an aperture of about $\left|\mathbf{k}_{\|}\right| / k_{0}=$ 0.4 , consistent with the notion that nonzero OAM implies a higher-order Bessel function as the radial profile. The (a)
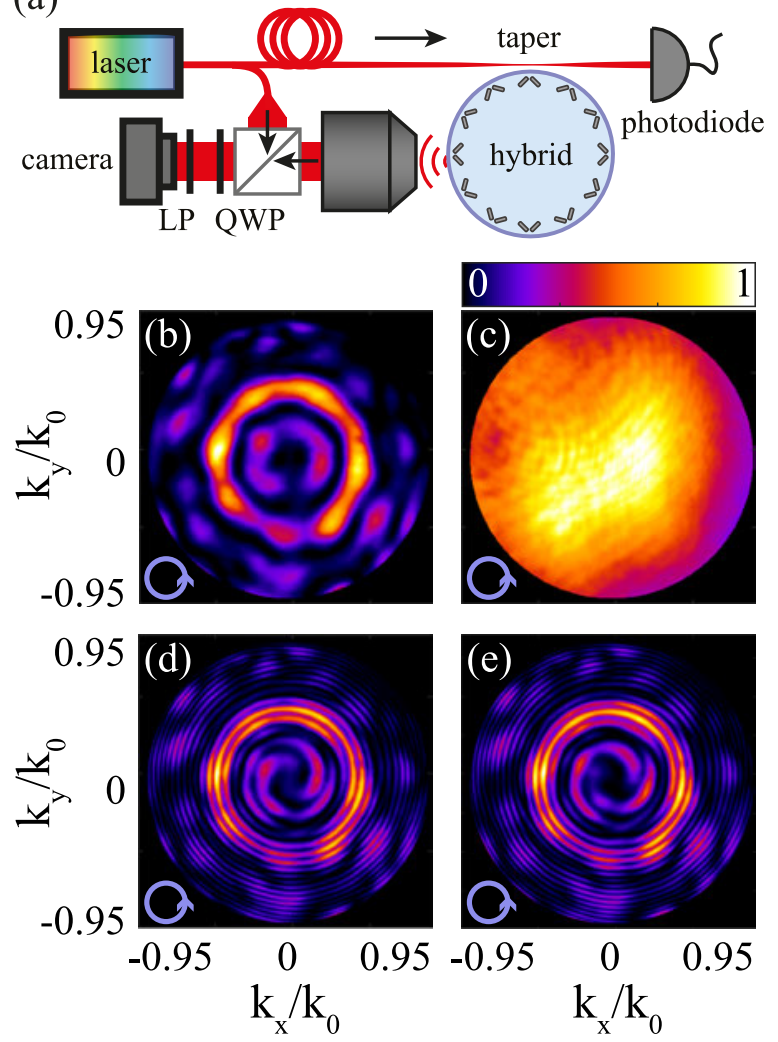

Figure 5. (a) Sketch of experimental setup. A narrowband tunable diode laser $(\lambda=765-781 \mathrm{~nm})$ is coupled into the antenna-dressed microdisk via a tapered fiber. The radiation pattern is collected from the top by a 0.95 NA objective, polarization analyzed using quarter wave plate (QWP) and linear polarizer (LP), and recombined with a reference beam to record interferometric Fourier images. (b-e) Raw experimental data: (b) Fourier image of the far-field intensity radiated in left-handed circular polarization (LHCP) by a cavity of radius 2 $\mu \mathrm{m}$, dressed by $N=19$ pairs of antennas for the V-type structure, at resonance for a WGM $m=22$ at $\lambda=772.5 \mathrm{~nm}$. (c) Fourier image of the intensity (in LHCP) of the reference beam used for interferometric phase retrieval. (d, e) Interferograms obtained when combining reference and analyzed beam for two different phase delays. The two-armed spiral confirms the generation of a beam of OAM $l=m-N-1=+2$ for LHCP.

interferograms show a distinct two-arm spiral structure that is characteristic for a beam carrying OAM of $l= \pm 2$ interfering with a reference beam of parabolic phase front. As we change the phase difference between the signal and reference beam, the spiral rotates around the center $\mathbf{k}_{\|} / k_{0}=0$ of the radiation pattern (compare Figure $5 \mathrm{~d}$ and Figure $5 \mathrm{e}$ ). The direction of rotation indicates the sign of the OAM. While the presence of a spiral in interferograms qualitatively indicates OAM, a full modal decomposition is required. This requires first reconstructing the full amplitude and phase from the interferograms, and subsequently calculation of the projection of this measured complex-valued field on OAM.

To reconstruct the required full phase and amplitude, we for each structure quantitatively analyze the sets of 200 interferograms per structure and per polarization channel using principal component analysis (PCA). ${ }^{67}$ We present in Figure 6 the complete analysis of such a data set: i.e., phase and intensity profiles of the scattered field in both circular polarization channels. Figure 6 reports data for the case of $m$ $=22$ and $N=20$, for three devices, i.e., for the radial-type 

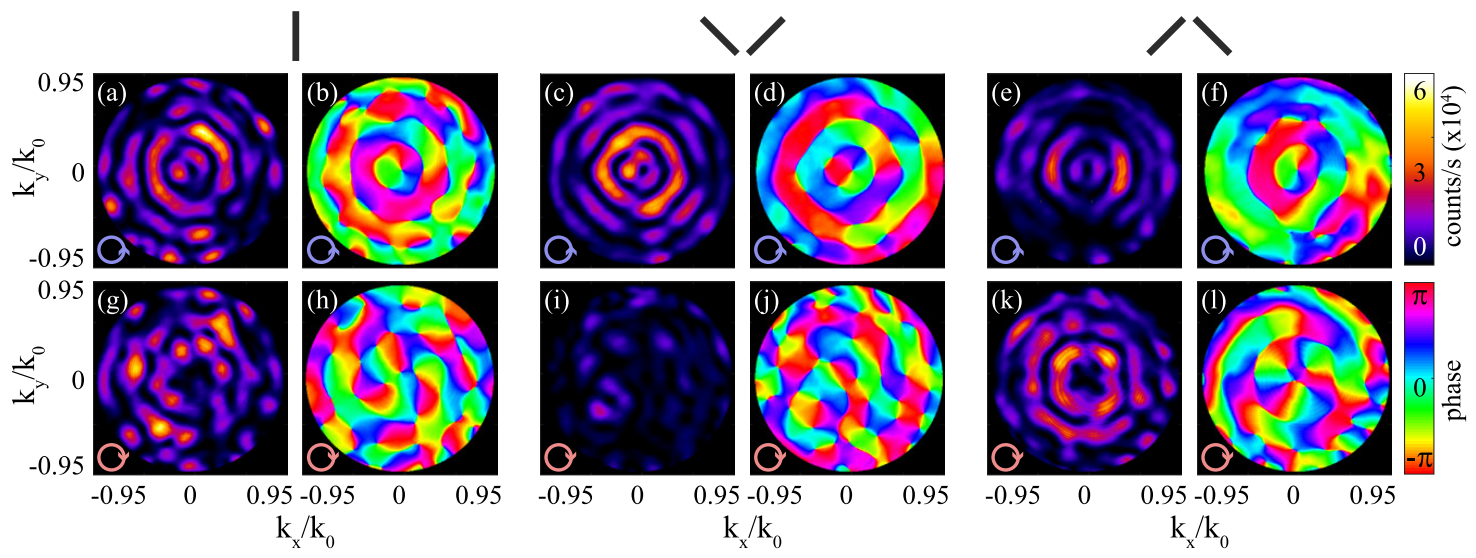

Figure 6. $k$-space distribution of intensity and phase distribution in the far field radiated by waveguide-driven devices with microdisk radius $2 \mu \mathrm{m}$, operating at WGM azimuthal quantum number $m=22$, and with $N=20$ unit cells, as reconstructed from interferometric Fourier microscopy. The top row of diagrams (panels a-f) refers to detection in the LHCP polarization channel, while the bottom row is for RHCP detection. Panels a and $\mathrm{g}$ and panels $\mathrm{b}$ and $\mathrm{h}$, respectively: intensity and concomitant phase for a device with radial antennas (single antenna per unit cell). Panels $\mathrm{c}$ and $\mathrm{i}$ and panels $\mathrm{d}$ and $\mathrm{j}$, respectively: intensity and phase for device with $\mathrm{V}$-type unit cells. Panels e and $\mathrm{k}$ and panels $\mathrm{f}$ and $\mathrm{l}$, respectively: intensity and concomitant phase maps for $\Lambda$-type structure. In all diagrams, the $x$ - and $y$-axes correspond to parallel momentum $\left(k_{x}, k_{y}\right) /=k_{0} \sin \theta(\cos \phi$, $\sin \phi)$ with $\theta$ and $\phi$ the polar and azimuthal angle in the radiation pattern, normalized to $k_{0}=\omega / c$. The images span the microscope NA of 0.95 . The intensity maps have a common color scale. The observations are similar to the calculated radiation pattern in Figure 4. However, for the right-hand circular polarized channel, the expected charge of $l_{\mathrm{R}}=m-N+1=+3$ is actually split into 3 charges +1 due to imperfections of our sample and measurements for panels $\mathrm{h}$ and $\mathrm{l}$. Due to the cancellation of the right-hand circular polarization by the V-type structure, we only observe a speckle noise pattern in panels $i$ and $j$.

(Figure 6a,b,g,h), V-type (Figure 6c,d,i,j), and $\Lambda$-type (Figure $6 \mathrm{e}, \mathrm{f}, \mathrm{k}, \mathrm{l})$ structures. The radial structure scatters as much light in left-handed circular polarization (LHCP) as in right-handed circular polarization (RHCP) (Figure 6a,g). On the other hand, the V-type structure clearly favors LHCP over RHCP, and we only observe weak speckle noise in the RHCP channel. The $\Lambda$-type structure dominantly outputs into RHCP over LHCP (Figure 6e,k), however, not canceling out LHCP completely down to a speckle noise level. Additionally, we note that the opening angle of the radiation pattern in the LHCP channel (Figure 6a,c,e) is smaller than for the RHCP channel (Figure 6g,i,k). This is commensurate with the notion that, for the considered geometry, the RHCP channel is expected to carry an OAM of $l_{\mathrm{R}}=m-N+1=+3$, higher than for LHCP with $l_{\mathrm{L}}=m-N-1=+1$. By analyzing the phase profiles, we indeed confirm that LHCP (Figure $6 \mathrm{~b}, \mathrm{~d}, \mathrm{f}$ ) exhibits a central phase singularity of charge equal to the expected OAM of $l_{\mathrm{L}}=+1$. Less evidently, for RHCP, Figure $6 \mathrm{~h}, 1$ suggests an OAM of +3 . However, closer inspection shows not a single singularity of charge +3 , but rather 3 singularities of charge +1 . These stem from the splitting of the single expected charge $l_{R}=+3$ and directly indicate that, likely due to sample and measurement imperfections, the radiation pattern suffers from the admixture of (small) terms that do not carry OAM = 3. Modal decomposition on an OAM basis is the tool of choice to quantify the finite purity of the radiation pattern that is only qualitatively evident from inspecting Figure 6.

We perform a modal decomposition of the measured complex-valued radiation diagrams. To this end, we employ the projection method reported by D'Errico ${ }^{68}$ for the general analysis of OAM content in beams. We first calculate the Fourier coefficients

$$
c_{l}(\nu)=\frac{1}{2 \pi} \int_{0}^{2 \pi} F_{s}(\nu, \phi) \mathrm{e}^{-i l \phi} \mathrm{d} \phi
$$

with respect to the azimuthal angular variable $\phi$, where $\nu \equiv$ $\left\|\mathbf{k}_{\|}\right\| / k_{0}$ and $F_{s}(\nu, \phi)$ indicate the measured amplitude and phase profile of the Fourier pattern. This overlap directly projects on OAM $l$ yet still depends on $k_{\|}$, i.e., on the radial dependence of the field that is evident in the data in Figure 6, which physically corresponds to the polar angle $\theta$ at which light is radiated. To obtain the OAM modal decomposition, we take the square modulus of $c_{l}$ and integrate out the radial dependence over the collection NA

$$
P_{l}=\int_{0}^{\mathrm{NA}}\left|c_{l}(\nu)\right|^{2} \mathrm{~d} \nu
$$

To illustrate the outcome of this modal decomposition procedure, Figure 7 reports histograms of OAM modal content $P_{l}$ for devices with $m-N=-1$, putting radial-type, V-type, and $\Lambda$-type structures alongside each other as Figure $7 \mathrm{a}-\mathrm{c}$, respectively. For each device, we measure LHCP and RHCP separately, which are separately reported (blue (red) columns at left (right) of pyramid plot for LHCP (RHCP)). Since the total radiated power is the sum of LHCP and RHCP, we report histograms of $P_{l}$ normalized to the sum of $P_{l}$ over all $l$ and polarization channels. This per-device procedure serves to normalize out differences in total radiated power between devices that occur due to variations in taper-cavity coupling strength. As expected, the radial antennas radiate approximately equal amounts in the LHCP $m-N-1$ and RHCP $m$ $-N+1$ channel, while the $\mathrm{V}$-type system is strongly biased to radiate only in the LHCP $m-N-1$ channels (93:7 ratio to the suppressed RHCP $m-N+1$ channel), while the $\Lambda$-type unit cell conversely favors the RHCP $m-N+1$. These histograms clearly make evident the expected OAM-spin selection rules. At the same time, it is clear that for all devices also all other OAM channels contain some radiation, without a clear preference for a particular OAM value. We attribute this background to imperfections in our sample and measurement. In particular any roughness and antenna size variation 


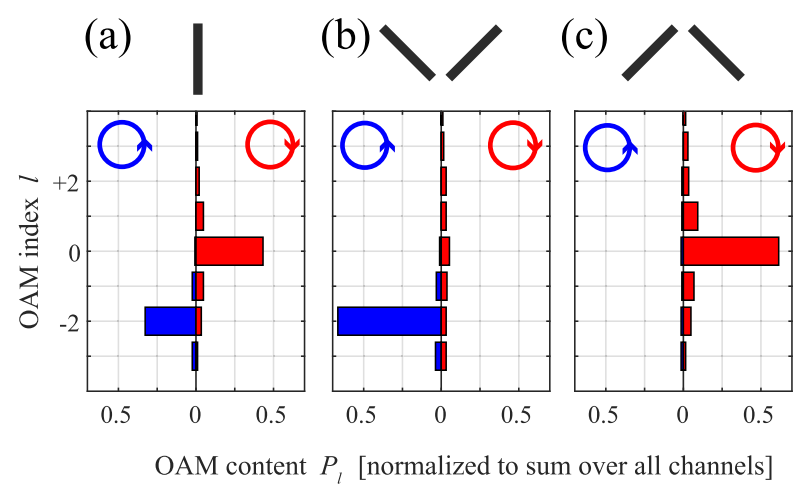

Figure 7. Modal decomposition diagram reporting OAM content $P_{l}$ (bar lengths, horizontal axes) versus OAM index $l$ (vertical axis) for radial-type, $\mathrm{V}$-type, and $\Lambda$-type structures as panels a-c, all with $m-$ $N=-1$. In each panel, the left-hand bars (blue) represent LHCP polarization and the right-hand bars (red) RHCP. The histograms are normalized such that for each device the sum of $P_{l}$ over all $l$ and both polarizations (sum of red and blue bars) is unity. The selection rule at $m-N=-1$ requires OAM $=-2$ and 0 , respectively, for LHCP and RHCP. In all three panels, the sum of these two contributions accounts for over $60 \%$ of radiation. The ratio between these two is a measure for the successful suppression of one OAM channel by unit cell design.

translates into random scattering, or equivalently into a speckle pattern which composes in a wide distribution of parasitic OAM contributions. The sum total of the desired OAM contribution relative to all parasitic terms is around 68:32 for the set of samples reported on in Figure 7.

To compare the performance in selectively generating OAM and polarization across many devices, we determine OAM modal decomposition histograms such as the one shown in Figure 7 for devices with varying $m-N$, spanning the range $-1,0, \ldots 3$ and encompassing radial-type, V-type, and $\Lambda$-type antennas (see the Supporting Information, Figure S4, for histograms for all devices). Figure 8 compiles the resulting histograms in a false color plot, so as to put devices with increasing $m-N$ alongside each other for direct comparison. In each false color plot, a single column represents a single device, with the OAM decomposition values $P_{l}$ for each device again normalized to their sum over all OAM and both polarizations. We plot the LHCP and RHCP detection channels separated into the top row (Figure $8 \mathrm{a}-\mathrm{c}$ ) and the bottom row (Figure $8 \mathrm{~d}-\mathrm{f}$ ) of false color plots. For the radial antenna case, systematically approximately equal amounts of LHCP and RHCP far field are generated, dominantly in OAM $m-N-1$ for LHCP and $m-N+1$ for RHCP as expected from the selection rule. These contributions are visible as the dominant diagonal features displaced vertically by $\mp 1$ from the red dashed diagonal that represents $l=N-m$. The ratio of these contributions is 50:50 \pm 10 averaged over devices (error reports standard deviation, i.e., variability between devices). The summed strength of these two contributions compared to the background that is due to sample imperfections is 69:31 \pm 6. For the V-type unit cell, the RHCP channel is strongly suppressed as expected, while the emission in the expected OAM $=m-N-1$ channel in LHCP is boosted. These channels stand in a 95:5 \pm 2 ratio averaged across devices, highlighting the excellent suppression by destructive interference with this unit cell design. The $\Lambda$-type antennas clearly favor right-handed polarization, although they are systematically less efficiently than the V-type structures with a 19:81 \pm

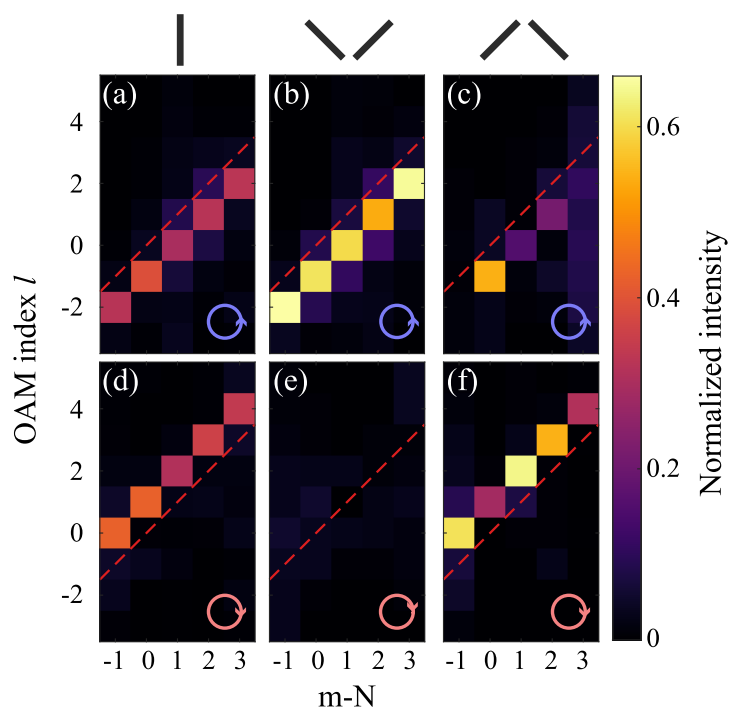

Figure 8. Measured modal decomposition onto OAM $l$ for microdisks (WGM azimuthal quantum number $m=22$ ) dressed by rings of nanoantennas with $N=19-23$ unit cells of radial I, V-type, and $\Lambda$-type designs. Panels a-c correspond to LHCP, while panels $\mathrm{d}-\mathrm{f}$ report on RHCP detection. (a, d) Radial I-unit cells, (b, e) V-type structures, and (c, f) $\Lambda$-type unit cells. In this representation, each single column represents an individual device, with the modal decomposition coefficient $P_{l}$ (see example Figure 7) given by the false color value. Each column is normalized to the sum over RHCP and LHCP and all OAM values. The dashed red lines indicate $l=m-N$. The data confirm the following OAM selection rules: (a) For radial antennas, the produced OAM is systematically equally distributed over $l_{\mathrm{L}}=m-N-1$ and $l_{\mathrm{R}}=m-N+1$. (b) The RHCP $l_{\mathrm{R}}=m-N+1$ channel is suppressed for $\mathrm{V}$ antennas, while the $l_{\mathrm{L}}=m-N-1$ channel is canceled in $\Lambda$-type structures.

12 ratio between OAM $m-N-1$ in LHCP and $m-N+1$ in RHCP. As is the case for the radial-antenna design, the performance is limited by disorder-induced scattering, with parasitic OAM channels accounting for between 30\% and $40 \%$ of radiation. This comparatively high number is the aggregate result of imperfections in the setup (speckle generated in optics downstream of the sample, as well as scattering in the fiber taper region that is in the microscope field of view, but away from the device) and actual device performance limits. For the devices themselves, we envision that particularly roughness of the dielectric covering the disks and size fluctuations in the antennas limit performance. Aside from improved fabrication strategies, a possible path to improvement could be to work with larger-radius structures, as increasing the number of unit cells could boost coherent addition into desired OAM channels over the effect of disorder. This strategy will, however, face a trade-off with the concomitant resulting smaller beam diameters: larger radii may cause higher-order OAM from the OAM-comb that is intrinsic to discrete OAM generators to appear more dominantly, and closer to $k=0$. Another parameter that would increase the ratio of OAM to undesirable background from, e.g., the taper region and cavity roughness is to increase the cavity-antenna coupling strength by reducing the taper thickness. From the perspective of waveguide-addressed OAM generation and detection, we note that it would be desirable to benchmark the overall efficiency from waveguide to far-field OAM. Unfortunately a manual fiber tapering approach prevents such a quantification. The desirable integration scenario would be to 
include the waveguide in the lithography, and to ensure that critical coupling is met. Since the cavity $Q$ is limited by coupling to the antennas, efficiencies at least as high as the antenna albedo should be reachable. Finally, we note that for the case $m=N$ one would not necessarily expect a strong polarization selectivity since in this case the degeneracy of the cavity mode is lifted, and the WGM modes split into standing waves, only one of which couples strongly to the antennas. The standing wave character means that the phase delay between the two antennas in a unit cell is no longer pinned at the value required for circular polarization. While Figure $8 \mathrm{c}-\mathrm{f}$ shows this feature for the $\Lambda$-antenna, it is surprising that the $\mathrm{V}$-antenna case in Figure $8 \mathrm{~b}-\mathrm{e}$ does not. We attribute this to fabrication imperfections in the particular device, which according to scanning electron microscope inspection happened to have a few deficient antennas, thereby breaking symmetry. For the interrogated cavity in Figure $8 c-f$, we verified with spectroscopic measurements that we indeed have a lifted degeneracy, with one high- $Q$ mode $\left(Q \approx 10^{4}\right)$ and a lower- $Q$ one $(Q \approx 800)$, while the case in Figure 8 b,e did not.

\section{CONCLUSION AND OUTLOOK}

In conclusion, we have demonstrated a new strategy for polarization-pure generation of single-OAM value beams by means of waveguide-addressed microdisk cavities. While OAM-pure output from microdisk cavities decorated with scattering units was demonstrated previously in microdisk lasing scenarios, these implementations rely on carefully designed spin-orbit coupling ${ }^{52}$ and are therefore not trivial to achieve. The main mechanism that we leverage is unit cell polarizability tensor engineering adapted to the driving WGM cavity field, loosely in the spirit of phase gradient metasurface unit cells. A limitation that is common to all methods that generates OAM from a discrete set of radiators is that the main OAM is accompanied by a comb of parasitic higher-order OAM values. If the number of antennas is high enough, these higher harmonics carry phase singularities of charges which are too high to be observed in the paraxial direction. In our experiment, fabrication disorder is the dominant limiting factor, instead of parasitic higher-order OAM values due to the discreteness of the antenna chain.

As an outlook, we envision that unit cell engineering provides many more degrees of freedom for engineering a rich set of vector beams. For instance, there is significant interest in vector vortex beams constructed as linear combinations of orthogonal, circularly polarized beams of different topological charges (OAM), ${ }^{69,70}$ of interest for, for instance, microscopy, optical forces, trapping and manipulation, and quantum optics with entangle single photons. Such beams are describable by higher-order Poincaré spheres (linear combinations of orthogonally circularly polarized beams of generally different OAM). The structures examined in this work could be generalized to realize particular subsets of this space of beams. For instance, linear combinations of orthogonally circularly polarized beams of opposite-sign OAM are available by addressing the waveguides from two ports simultaneously. One can further envision multiplexing rings of different numbers of antennas on a single disk, as well as inserting twists from unit cell to unit cell in the antenna orientation. We envision that such schemes could be used to dynamically tune vectors beams, e.g., in sorting applications for tiny particles or enantiomers, through the coherently controlled waveguide addressing of microdisk-antenna units.

\section{METHODS}

Nanofabrication. We used a two-step electron-beam lithography method to realize $\mathrm{Si}_{3} \mathrm{~N}_{4}$ disks, hybridized with aluminum antennas placed on top of the cavity. ${ }^{56,71}$ First, we fabricate $\mathrm{Si}_{3} \mathrm{~N}_{4}\left(n_{\mathrm{Si}_{3} \mathrm{~N}_{4}} \approx 2.0\right)$ microdisks on silicon pedestals. Our fabrication is limited to a fixed $200 \mathrm{~nm}$ thickness, and we choose diameters in a small range around $4 \mu \mathrm{m}$ diameter to target $m \sim 20-25$ azimuthal mode numbers. We evaporate a $110 \mathrm{~nm}$ glass spacer $\left(n_{\text {glass }} \approx 1.5\right)$ on top of the disk to lift the antennas out of the cavity mode, thereby reducing the $Q$ deteriorating perturbation strength of our arrays of antennas. The optimum placement in TE modes of the fundamental radial is at $230 \mathrm{~nm}$ from the edge of the disk (cf. Supporting Information). Antennas are made by lift-off, using thermally evaporated aluminum to obtain $\approx 140 \mathrm{~nm}$ long, $\approx 30 \mathrm{~nm}$ thick, and $\approx 40 \mathrm{~nm}$ wide antennas. Such antennas are expected to be near-resonant to allow for a large polarizability anisotropy $\left|\alpha_{\text {long }} / \alpha_{\text {short }}\right|^{2}>100$, but somewhat blue-detuned from our cavities operating at $765-781 \mathrm{~nm}$ to mitigate the broadening of cavity resonances (full wave calculations with optical constants from ref 72 described in the Supporting Information). Using scanning electron micrographs (SEMs), we quantify an excellent yield of antennas present on the sample after fabrication above $97.5 \%$ (cf. Figure 1c,d).

Interferometric Fourier Microscopy. We use tapered fiber excitation (Corning $\mathrm{HI} 780 \mathrm{C}$, pulled to a taper on a $\mathrm{H}_{2}$ flame) and perform high-NA (NA $=0.95)$ phase-resolved Fourier imaging of the radiation pattern as shown in Figure 5a. A New Focus Velocity diode laser operating at 765-781 nm ensures that we can tune to specific WGM modes. The light collected through an IR objective of NA $=0.95$ is recombined with a reference beam onto a circular polarization analyzer consisting of a quarter wave plate, followed by a linear polarizer. The interferogram is then recorded by a CCD camera. The reference beam has a relatively homogeneous intensity over the camera, but a parabolic phase front of which we align the center approximately to the center of the objective NA. For phase fronts with pure OAM, the interferogram should therefore exhibit spirals with the number of arms equal to the OAM of the investigated beam. ${ }^{36}$ While one could extract phase from a single interferogram by digital off-axis holography, ${ }^{32,68,73}$ we instead use on-axis interferometry. We measure a set of interferograms with varying phase differences between reference and analyzed beams.

To this end, we unbalanced the interferometer arms in the optical path length, so that slight variations of the excitation wavelength $(<1 \mathrm{pm}$, much less than the dressed cavity line width) vary the reference phase. An optical path difference of order $50-60 \mathrm{~cm}$ yields a $6 \pi$ phase shift for $\delta \lambda=0.5 \mathrm{pm}$ at a wavelength of $\lambda=780 \mathrm{~nm}$. For each measurement sequence, our protocol is to first find the resonance of the dressed cavities in the scattering spectrum. Then, we set the circular polarization analyzer to left-handed polarization, measure the intensity of the emitted beam and reference separately, and then recombine the two beams and record 200 interferograms with a phase delay varied from 0 to $6 \pi$ ( 3 fringes). We then repeat the procedure for right-handed polarization.

\section{ASSOCIATED CONTENT}

\section{sI Supporting Information}

The Supporting Information is available free of charge at https://pubs.acs.org/doi/10.1021/acsphotonics.0c01081. 
More extensive mode analysis of microdisks, details of the full-wave numerical design study, and OAM efficiency histograms for all devices (PDF)

\section{AUTHOR INFORMATION}

\section{Corresponding Author}

A. Femius Koenderink - Center for Nanophotonics, AMOLF, 1098 XG Amsterdam, The Netherlands; Van der WaalsZeeman Institute, University of Amsterdam, 1090 GL Amsterdam, The Netherlands; 이이.org/0000-0003-16175748; Email: f.koenderink@amolf.nl

\section{Authors}

Kévin G. Cognée - Center for Nanophotonics, AMOLF, 1098 XG Amsterdam, The Netherlands; LP2N, Institut d'Optique Graduate School, CNRS, Univ. Bordeaux, 33400 Talence, France

Hugo M. Doeleman - Center for Nanophotonics, AMOLF, 1098 XG Amsterdam, The Netherlands; Van der WaalsZeeman Institute, University of Amsterdam, 1090 GL Amsterdam, The Netherlands; 이이. orcid.org/0000-0003-29768294

Philippe Lalanne - LP2N, Institut d'Optique Graduate School, CNRS, Univ. Bordeaux, 33400 Talence, France; 이이. ord.org/ 0000-0003-1979-2290

Complete contact information is available at:

https://pubs.acs.org/10.1021/acsphotonics.0c01081

\section{Notes}

The authors declare no competing financial interest.

\section{ACKNOWLEDGMENTS}

The authors thank the AMOLF Nanolab support team, Isabelle Palstra and Annemarie Berkhout with help in fabrication, John Mathew and Ewold Verhagen for encouragement and use of the fiber tapering setup, and Ruslan Röhrich and Radoslaw Kolkowski for the technical and artistic contributions. This work is part of the research programme Hybrid nanophotonic architectures for ultrafast quantum optics [NWO-Vici] with Project 680.47 .621 , which is financed by the Dutch Research Council (NWO), and the "Investments for the Future" program of IdEx Bordeaux. The research was performed at the NWO research institute AMOLF.

\section{REFERENCES}

(1) Poynting, J. H. The Wave Motion of a Revolving Shaft, and a Suggestion as to the Angular Momentum in a Beam of Circularly Polarised Light. Proc. R. Soc. London, Ser. A 1909, 82, 560-567.

(2) Beth, R. A. Mechanical Detection and Measurement of the Angular Momentum of Light. Phys. Rev. 1936, 50, 115-125.

(3) Condon, E. U.; Shortley, G. H. The Theory of Atomic Spectra; Cambridge University Press: Cambridge, UK, 1935.

(4) Allen, L.; Beijersbergen, M. W.; Spreeuw, R. J. C.; Woerdman, J. P. Orbital Angular Momentum of Light and the Transformation of Laguerre-Gaussian Laser Modes. Phys. Rev. A: At., Mol., Opt. Phys. 1992, 45, 8185-8189.

(5) Coullet, P.; Gil, L.; Rocca, F. Optical vortices. Opt. Commun. 1989, 73, 403-408.

(6) Willig, K. I.; Rizzoli, S. O.; Westphal, V.; Jahn, R.; Hell, S. W. STED Microscopy Reveals That Synaptotagmin Remains Clustered after Synaptic Vesicle Exocytosis. Nature 2006, 440, 935-939.

(7) Lee, J. H.; Foo, G.; Johnson, E. G.; Swartzlander, G. A. Experimental Verification of an Optical Vortex Coronagraph. Phys. Rev. Lett. 2006, 97, 053901.
(8) Rodríguez-Herrera, O. G.; Lara, D.; Bliokh, K. Y.; Ostrovskaya, E. A.; Dainty, C. Optical Nanoprobing via Spin-Orbit Interaction of Light. Phys. Rev. Lett. 2010, 104, 253601.

(9) Aleksanyan, A.; Brasselet, E. Self-Eclipsing: Alignment-Free Vortex Coronagraphy. Opt. Lett. 2017, 42, 1237.

(10) Grier, D. G. A. Revolution in Optical Manipulation. Nature 2003, 424, 810-816.

(11) Padgett, M.; Bowman, R. Tweezers with a Twist. Nat. Photonics 2011, 5, 343-348.

(12) Dholakia, K.; Čižmár, T. Shaping the Future of Manipulation. Nat. Photonics 2011, 5, 335-342.

(13) Woerdemann, M.; Alpmann, C.; Esseling, M.; Denz, C. Advanced Optical Trapping by Complex Beam Shaping. Laser Photonics Rev. 2013, 7, 839-854.

(14) Willner, A. E.; et al. Optical Communications Using Orbital Angular Momentum Beams. Adv. Opt. Photonics 2015, 7, 66.

(15) Ghadimi Nassiri, M.; Brasselet, E. Multispectral Management of the Photon Orbital Angular Momentum. Phys. Rev. Lett. 2018, 121, 213901.

(16) Fickler, R.; Campbell, G.; Buchler, B.; Lam, P. K.; Zeilinger, A. Quantum Entanglement of Angular Momentum States with Quantum Numbers Up to 10,010. Proc. Natl. Acad. Sci. U. S. A. 2016, 113, 13642-13647.

(17) Erhard, M.; Fickler, R.; Krenn, M.; Zeilinger, A. Twisted Photons: New Quantum Perspectives in High Dimensions. Light: Sci. Appl. 2018, 7, 17146.

(18) Vuong, L. T.; Adam, A. J.; Brok, J. M.; Planken, P. C.; Urbach, H. P. Electromagnetic Spin-Orbit Interactions via Scattering of Subwavelength Apertures. Phys. Rev. Lett. 2010, 104, 083903.

(19) Marrucci, L.; Manzo, C.; Paparo, D. Optical Spin-To-Orbital Angular Momentum Conversion in Inhomogeneous Anisotropic Media. Phys. Rev. Lett. 2006, 96, 163905.

(20) Bliokh, K. Y.; Rodríguez-Fortuño, F. J.; Nori, F.; Zayats, A. V. Spin-Orbit Interactions of Light. Nat. Photonics 2015, 9, 796-808.

(21) Gorodetski, Y.; Drezet, A.; Genet, C.; Ebbesen, T. W. Generating Far-Field Orbital Angular Momenta from Near-Field Optical Chirality. Phys. Rev. Lett. 2013, 110, 203906.

(22) Lodahl, P.; Mahmoodian, S.; Stobbe, S.; Rauschenbeutel, A.; Schneeweiss, P.; Volz, J.; Pichler, H.; Zoller, P. Chiral Quantum Optics. Nature 2017, 541, 473-480.

(23) Alpeggiani, F.; Bliokh, K. Y.; Nori, F.; Kuipers, L. Electromagnetic Helicity in Complex Media. Phys. Rev. Lett. 2018, 120, 243605.

(24) Shen, Y.; Wang, X.; Xie, Z.; Min, C.; Fu, X.; Liu, Q.; Gong, M.; Yuan, $\mathrm{X}$. Optical Vortices 30 years on: OAM manipulation from topological charge to multiple singularities. Light: Sci. Appl. 2019, 8, 90.

(25) Yao, A.; Padgett, M. Orbital angular momentum: origins, behavior and applications. Adv. Opt. Photonics 2011, 3, 161-204.

(26) Brasselet, E.; Malinauskas, M.; Žukauskas, A.; Juodkazis, S. Photopolymerized Microscopic Vortex Beam Generators: Precise Delivery of Optical Orbital Angular Momentum. Appl. Phys. Lett. 2010, 97, 211108.

(27) Cheong, W. C.; Lee, W. M.; Yuan, X.-C.; Zhang, L.-S.; Dholakia, K.; Wang, H. Direct Electron-Beam Writing of Continuous Spiral Phase Plates in Negative Resist with High Power Efficiency for Optical Manipulation. Appl. Phys. Lett. 2004, 85, 5784-5786.

(28) Oemrawsingh, S. S. R.; van Houwelingen, J. A. W.; Eliel, E. R.; Woerdman, J. P.; Verstegen, E. J. K.; Kloosterboer, J. G.; 't Hooft, G. W. Production and Characterization of Spiral Phase Plates for Optical Wavelengths. Appl. Opt. 2004, 43, 688.

(29) Curtis, J. E.; Koss, B. A.; Grier, D. G. Dynamic Holographic Optical Tweezers. Opt. Commun. 2002, 207, 169-175.

(30) Brasselet, E.; Loussert, C. Electrically Controlled Topological Defects in Liquid Crystals as Tunable Spin-Orbit Encoders for Photons. Opt. Lett. 2011, 36, 719.

(31) Caño-García, M.; Quintana, X.; Otón, J. M.; Geday, M. A. Dynamic Multilevel Spiral Phase Plate Generator. Sci. Rep. 2018, 8, 15804. 
(32) Röhrich, R.; Hoekmeijer, C.; Osorio, C. I.; Koenderink, A. F. Quantifying Single Plasmonic Nanostructure Far-Fields with Interferometric and Polarimetric K-Space Microscopy. Light: Sci. Appl. 2018, 7, 65 .

(33) Sharma, D. K.; Kumar, V.; Vasista, A. B.; Paul, D.; Chaubey, S. K.; Pavan Kumar, G. V. Optical Orbital Angular Momentum ReadOut Using a Self-Assembled Plasmonic Nanowire. ACS Photonics 2019, 6, 148-153.

(34) Kerber, R. M.; Fitzgerald, J. M.; Reiter, D. E.; Oh, S. S.; Hess, O. Reading the Orbital Angular Momentum of Light Using Plasmonic Nanoantennas. ACS Photonics 2017, 4, 891-896.

(35) Kerber, R. M.; Fitzgerald, J. M.; Oh, S. S.; Reiter, D. E.; Hess, O. Orbital Angular Momentum Dichroism in Nanoantennas. Commun. Phys. 2018, 1, 87.

(36) Devlin, R. C.; Ambrosio, A.; Wintz, D.; Oscurato, S. L.; Zhu, A. Y.; Khorasaninejad, M.; Oh, J.; Maddalena, P.; Capasso, F. Spin-ToOrbital Angular Momentum Conversion in Dielectric Metasurfaces. Opt. Express 2017, 25, 377.

(37) Wang, J.; Yang, J. Y.; Fazal, I. M.; Ahmed, N.; Yan, Y.; Huang, H.; Ren, Y.; Yue, Y.; Dolinar, S.; Tur, M.; Willner, A. E. Terabit FreeSpace Data Transmission Employing Orbital Angular Momentum Multiplexing. Nat. Photonics 2012, 6, 488-496.

(38) Chong, K. E.; Staude, I.; James, A.; Dominguez, J.; Liu, S.; Campione, S.; Subramania, G. S.; Luk, T. S.; Decker, M.; Neshev, D. N.; Brener, I.; Kivshar, Y. S. Polarization-Independent Silicon Metadevices for Efficient Optical Wavefront Control. Nano Lett. 2015, 15, 5369-5374.

(39) Yang, Y.; Wang, W.; Moitra, P.; Kravchenko, I. I.; Briggs, D. P.; Valentine, J. Dielectric Meta-Reflectarray for Broadband Linear Polarization Conversion and Optical Vortex Generation. Nano Lett. 2014, 14, 1394-1399.

(40) Sun, J.; Wang, X.; Xu, T.; Kudyshev, Z. A.; Cartwright, A. N.; Litchinitser, N. M. Spinning Light on the Nanoscale. Nano Lett. 2014, 14, 2726-2729.

(41) Hakobyan, D.; Magallanes, H.; Seniutinas, G.; Juodkazis, S.; Brasselet, E. Tailoring Orbital Angular Momentum of Light in the Visible Domain with Metallic Metasurfaces. Adv. Opt. Mater. 2016, 4, 306-312.

(42) Miao, P.; Zhang, Z.; Sun, J.; Walasik, W.; Longhi, S.; Litchinitser, N. M.; Feng, L. Orbital Angular Momentum Microlaser. Science 2016, 353, 464-467.

(43) Zhang, J.; et al. An InP-Based Vortex Beam Emitter with Monolithically Integrated Laser. Nat. Commun. 2018, 9, 2652.

(44) Mock, A.; Sounas, D.; Alù, A. Tunable Orbital Angular Momentum Radiation from Angular-Momentum-Biased Microcavities. Phys. Rev. Lett. 2018, 121, 103901.

(45) Hayenga, W. E.; Parto, M.; Ren, J.; Wu, F. O.; Hokmabadi, M. P.; Wolff, C.; El-Ganainy, R.; Mortensen, N. A.; Christodoulides, D. N.; Khajavikhan, M. Direct Generation of Tunable Orbital Angular Momentum Beams in Microring Lasers with Broadband Exceptional Points. ACS Photonics 2019, 6, 1895-1901.

(46) Seghilani, M.; Myara, M.; Sellahi, M.; Legratiet, L.; Sagnes, I.; Beaudoin, G.; Lalanne, P.; Garnache, A. Vortex Laser Based on III-V Semiconductor Metasurface: Direct Generation of Coherent Laguerre- Gauss Modes Carrying Controlled Orbital Angular Momentum. Sci. Rep. 2016, 6, 38156.

(47) Carlon Zambon, N.; St-Jean, P.; Milićević, M.; Lemaître, A.; Harouri, A.; Le Gratiet, L.; Bleu, O.; Solnyshkov, D. D.; Malpuech, G.; Sagnes, I.; Ravets, S.; Amo, A.; Bloch, J. Optically Controlling the Emission Chirality of Microlasers. Nat. Photonics 2019, 13, 283-288.

(48) Cai, X. X.-L.; Wang, J.-W. U. o. B. J.; Strain, M. J.; JohnsonMorris, B.; Zhu, J.-B. J.; Sorel, M.; O’Brien, J. L.; Thompson, M. G.; Yu, S. S.-Y. Integrated Compact Optical Vortex Beam Emitters. Science 2012, 338, 363-366.

(49) Wang, Y.; Feng, X.; Zhang, D.; Zhao, P.; Li, X.; Cui, K.; Liu, F.; Huang, Y. Generating Optical Superimposed Vortex Beam with Tunable Orbital Angular Momentum Using Integrated Devices. Sci. Rep. 2015, 5, 10958.
(50) Xiao, Q.; Klitis, C.; Li, S.; Chen, Y.; Cai, X.; Sorel, M.; Yu, S. Generation of Photonic Orbital Angular Momentum Superposition States Using Vortex Beam Emitters with Superimposed Gratings. Opt. Express 2016, 24, 3168-3176.

(51) Rui, G.; Gu, B.; Cui, Y.; Zhan, Q. Detection of Orbital Angular Momentum Using a Photonic Integrated Circuit. Sci. Rep. 2016, 6, 28262.

(52) Shao, Z.; Zhu, J.; Chen, Y.; Zhang, Y.; Yu, S. Spin-Orbit Interaction of Light Induced by Transverse Spin Angular Momentum Engineering. Nat. Commun. 2018, 9, 926.

(53) Rosenthal, J. E.; Murphy, G. M. Group Theory and the Vibrations of Polyatomic Molecules. Rev. Mod. Phys. 1936, 8, 317346.

(54) Mirin, N. A.; Bao, K.; Nordlander, P. Fano Resonances in Plasmonic Nanoparticle Aggregates. J. Phys. Chem. A 2009, 113, $4028-4034$

(55) Jen, H. H.; Chang, M.-S.; Chen, Y.-C. Cooperative Light Scattering from Helical-Phase-Imprinted Atomic Rings. Sci. Rep. 2018, 8, 9570.

(56) Cognée, K. G.; Doeleman, H. M.; Lalanne, P.; Koenderink, A. F. Cooperative Interactions between Nanoantennas in a High $Q$ Cavity for Unidirectional Light Sources. Light: Sci. Appl. 2019, 8, 115.

(57) Yang, J.; Giessen, H.; Lalanne, P. Simple Analytical Expression for the Peak-Frequency Shifts of Plasmonic Resonances for Sensing. Nano Lett. 2015, 15, 3439-3444.

(58) Ruesink, F.; Doeleman, H. M.; Hendrikx, R.; Koenderink, A. F.; Verhagen, E. Perturbing Open Cavities: Anomalous Resonance Frequency Shifts in a Hybrid Cavity-Nanoantenna System. Phys. Rev. Lett. 2015, 115, 203904.

(59) Pan, F.; Smith, K. C.; Nguyen, H. L.; Knapper, K. A.; Masiello, D. J.; Goldsmith, R. H. Elucidating Energy Pathways through Simultaneous Measurement of Absorption and Transmission in a Coupled Plasmonic-Photonic Cavity. Nano Lett. 2020, 20, 50-58.

(60) Thakkar, N.; Rea, M. T.; Smith, K. C.; Heylman, K. D.; Quillin, S. C.; Knapper, K. A.; Horak, E. H.; Masiello, D. J.; Goldsmith, R. H. Sculpting Fano Resonances To Control Photonic-Plasmonic Hybridization. Nano Lett. 2017, 17, 6927-6934.

(61) Bozzola, A.; Perotto, S.; De Angelis, F. Hybrid plasmonicphotonic whispering gallery mode resonators for sensing: a critical review. Analyst 2017, 142, 883-898.

(62) Novotny, L.; Hecht, B. Principles of Nano-Optics; Cambridge University Press, 2006.

(63) Lalanne, P.; Yan, W.; Vynck, K.; Sauvan, C.; Hugonin, J.-P. Light Interaction with Photonic and Plasmonic Resonances. Laser Photonics Rev. 2018, 12, 1700113.

(64) Cognée, K. G.; Yan, W.; La China, F.; Balestri, D.; Intonti, F.; Gurioli, M.; Koenderink, A. F.; Lalanne, P. Mapping Complex Mode Volumes with Cavity Perturbation Theory. Optica 2019, 6, 269.

(65) Zhu, J.; Cai, X.; Chen, Y.; Yu, S. Theoretical Model for Angular Grating-Based Integrated Optical Vortex Beam Emitters. Opt. Lett. 2013, 38, 1343.

(66) Ding, F.; Pors, A.; Bozhevolnyi, S. I. Gradient Metasurfaces: a Review of Fundamentals and Applications. Rep. Prog. Phys. 2018, 81, 026401.

(67) Vargas, J.; Quiroga, J. A.; Belenguer, T. Phase-Shifting Interferometry Based on Principal Component Analysis. Opt. Lett. 2011, 36, 1326

(68) D’Errico, A.; D’Amelio, R.; Piccirillo, B.; Cardano, F.; Marrucci, L. Measuring the Complex Orbital Angular Momentum Spectrum and Spatial Mode Decomposition of Structured Light Beams. Optica 2017, 4, 1350.

(69) Milione, G.; Sztul, H. I.; Nolan, D. A.; Alfano, R. R. HigherOrder Poincaré Sphere, Stokes Parameters, and the Angular Momentum of Light. Phys. Rev. Lett. 2011, 107, 053601.

(70) Yi, X.; Liu, Y.; Ling, X.; Zhou, X.; Ke, Y.; Luo, H.; Wen, S.; Fan, D. Hybrid-Order PoincarE Sphere. Phys. Rev. A: At., Mol., Opt. Phys. 2015, 91, 023801.

(71) Doeleman, H. M. Hybrid Resonators for Light Trapping and Emission Control. Ph.D. thesis, University of Amsterdam, 2019. 
(72) Rakić, A. D.; Djurǐ̌ić, A. B.; Elazar, J. M.; Majewski, M. L. Optical Properties of Metallic Films for Vertical-Cavity Optoelectronic Devices. Appl. Opt. 1998, 37, 5271.

(73) Kulkarni, G.; Sahu, R.; Magaña-Loaiza, O. S.; Boyd, R. W.; Jha, A. K. Single-Shot Measurement of the Orbital-Angular-Momentum Spectrum of Light. Nat. Commun. 2017, 8, 1054. 